\title{
Normal families of meromorphic functions which share a set
}

\author{
Jinhua Cai, Fanning Meng, Jia Xie, Wenjun Yuan* \\ School of Mathematics and Information Sciences, Guangzhou University, Guangzhou 510006 China \\ *Corresponding author, e-mail: wjyuan1957@126.com
}

Received 8 Jul 2019

Accepted 9 Apr 2020

\begin{abstract}
In this paper, by using the Nevanlinna's value distribution theory and the method of Zalcman-Pang, it investigates the normality of a family of meromorphic functions, denoted by $\mathscr{F}$, defined in a domain $D$, which concerns the conditions for each $f \in \mathscr{F}$ : (i) $\bar{E}\left(S_{1}, f\right)=\bar{E}\left(S_{2},\left(f^{(k)}\right)^{q}\right)$; (ii) both zeros and poles of $f-a$ have multiplicities at least $k(>2$ or $\geqslant 2)$ and $k+1$, respectively, or $k(\geqslant 4)$ and $k-1$, respectively, where $k$ and $q$ are positive integers, $a$ is any finite complex number, $S_{1}=\left\{a_{1}, a_{2}, a_{3}\right\}$ and $S_{2}=\left\{b_{1}, b_{2}, b_{3}\right\}$ are made up of finite complex numbers. The conclusion still holds if condition (ii) is replaced by the assumption that zeros of $f-a_{i}$ have multiplicities at least $k$, where $k \geqslant 1$ and $i=1,2,3$.
\end{abstract}

KEYWORDS: meromorphic functions, normality, shared set

MSC2010: 30D35 30D45

\section{INTRODUCTION}

In this paper, we use the standard notations and definitions of values distribution theory such as $T(r, f), N(r, f), \bar{N}(r, f)$ and $S(r, f)$, see $[1,2]$.

Let $D$ be a domain in $\mathbb{C}$ and let $\mathscr{F}$ be a family of meromorphic functions defined in $D$. $\mathscr{F}$ is said to be normal in $D$ in the sense of Montel if each sequence $\left\{f_{n}\right\} \subset \mathscr{F}$ contains a subsequence that converges spherically locally uniformly in $D$, to a meromorphic function or $\infty$, see $[3,4]$.

Let $f$ and $g$ be two meromorphic functions in a domain $D$, and let $a$ be any complex number in $\mathbb{C}$. If the zeros of $f-a$ are the same as the zeros of $g-a$, we say $f$ and $g$ share $a \mathrm{IM}$, see $[5,6]$.

Let $\mathscr{F}$ be a family of meromorphic functions in a domain $D$, if there exists a neighborhood (denoted by $\Delta\left(z_{0}\right)$ ) of point $z_{0}$ such that $\mathscr{F}$ is normal in $\Delta\left(z_{0}\right)$, $\mathscr{F}$ is said to be normal at $z_{0} \in D$,see $[7,8]$.

Schwick [9] was the first one who gave a connection between normality and shared values, and proved the following result.

Theorem A ([9]) Let $\mathscr{F}$ be a family of meromorphic functions in a domain $D$, let $a_{1}, a_{2}$ and $a_{3}$ be three distinct finite complex numbers. If $f$ and $f^{\prime}$ share $a_{i}$ IM for each $f \in \mathscr{F}$, where $i=1,2,3$, then $\mathscr{F}$ is normal in $D$.

Fang [10] generalized Theorem A as follows, and proposed the concept of shared set.
Let $f$ and $g$ be two meromorphic functions in a domain $D$, and let $S=\left\{a_{1}, a_{2}, a_{3}\right\}$, where $S$ consists of finite complex numbers. Denote $\bar{E}(S, f)=$ $\bigcup_{a_{i} \in S}\left\{z \in D: f(z)-a_{i}=0\right\}$, where $i=1,2,3$. If $\bar{E}(S, f)=\bar{E}(S, g)$, we say that $f$ and $g$ share $S$.

Theorem B ([10]) Let $\mathscr{F}$ be a family of holomorphic functions in a domain $D$, let $a_{1}, a_{2}$ and $a_{3}$ be three distinct finite complex numbers. If $\bar{E}(S, f)=\bar{E}\left(S, f^{\prime}\right)$ for any $f \in \mathscr{F}$, where $S=\left\{a_{1}, a_{2}, a_{3}\right\}$, then $\mathscr{F}$ is normal in $D$.

Since then, many results of normality criteria concerning sharing values have been obtained, for example in $[11,12]$. It is natural to ask whether the result is valid or not if $a_{1}, a_{2}$ and $a_{3}$ (Theorem A) are replaced by a set $S:=\left\{a_{1}, a_{2}, a_{3}\right\}$. In this direction, Liu and Pang [13] proved the following result.

Theorem C ([13]) Let $\mathscr{F}$ be a family of meromorphic functions in a domain $D$, let $a_{1}, a_{2}$ and $a_{3}$ be three distinct finite complex numbers. If $\bar{E}(S, f)=$ $\bar{E}\left(S, f^{\prime}\right)$ for any $f \in \mathscr{F}$, where $S=\left\{a_{1}, a_{2}, a_{3}\right\}$, then $\mathscr{F}$ is normal in $D$.

Zhang et al [14] showed the following result.

Theorem D ([14]) Let $\mathscr{F}$ be a family of meromorphic functions in a domain $D$, let $a_{1}, a_{2}$ and $a_{3}$ be three distinct finite complex numbers, let $k>2$ be a positive integer, let $a$ be any finite complex number, and let $S=\left\{a_{1}, a_{2}, a_{3}\right\}$. If for each $f \in \mathscr{F}$, 
(i) $\bar{E}(S, f)=\bar{E}\left(S, f^{(k)}\right)$;

(ii) both zeros and poles of $f-a$ are of multiplicities at least $k$,

then $\mathscr{F}$ is normal in $D$.

We generalize Theorem D as follows.

Theorem 1 Let $\mathscr{F}$ be a family of meromorphic functions in a domain $D$, let $S_{1}=\left\{a_{1}, a_{2}, a_{3}\right\}, S_{2}=$ $\left\{b_{1}, b_{2}, b_{3}\right\}$, where $S_{1}$ and $S_{2}$ are made up of finite complex numbers, let $k>2$ and $q$ be two positive integers, and let $a$ be any finite complex number. Suppose that for each $f \in \mathscr{F}$,

(i) $\bar{E}\left(S_{1}, f\right)=\bar{E}\left(S_{2},\left(f^{(k)}\right)^{q}\right)$;

(ii) both zeros and poles of $f-a$ have multiplicities at least $k$,

then $\mathscr{F}$ is normal in $D$.

In Theorem 1 , if condition (ii) is replaced by $f-$ $a_{i}(i=1,2,3)$ has zeros with multiplicities at least $k \geqslant 1$ or $f-a$ has zeros and poles with multiplicities at least $k \geqslant 2$ and $k+1$, respectively, or $f-a$ has zeros and poles with multiplicities at least $k \geqslant 4$ and $k-1$, respectively, the conclusion still holds. We get the following results.

Theorem 2 Let $\mathscr{F}$ be a family of meromorphic functions in a domain $D$, let $S_{1}=\left\{a_{1}, a_{2}, a_{3}\right\}, S_{2}=$ $\left\{b_{1}, b_{2}, b_{3}\right\}$, where $S_{1}$ and $S_{2}$ consist of finite complex numbers, and let $k$ and $q$ be two positive integers. If for all $f \in \mathscr{F}$,

(i) $\bar{E}\left(S_{1}, f\right)=\bar{E}\left(S_{2},\left(f^{(k)}\right)^{q}\right)$;

(ii) $f-a_{i}, i=1,2,3$, has zeros with multiplicities at least $k$,

then $\mathscr{F}$ is normal in $D$.

Remark 1 Theorem C is a corollary of Theorem 2.

Theorem 3 Let $\mathscr{F}$ be a family of meromorphic functions in a domain $D$, let $S_{1}=\left\{a_{1}, a_{2}, a_{3}\right\}, S_{2}=$ $\left\{b_{1}, b_{2}, b_{3}\right\}$, where $S_{1}$ and $S_{2}$ consist of finite complex numbers, let $k \geqslant 2$ and $q$ be two positive integers, and let a be any finite complex number. If for each $f \in \mathscr{F}$, (i) $\bar{E}\left(S_{1}, f\right)=\bar{E}\left(S_{2},\left(f^{(k)}\right)^{q}\right)$;

(ii) $f-a$ has zeros and poles with multiplicities at least $k$ and $k+1$, respectively,

then $\mathscr{F}$ is normal in $D$.

Theorem 4 Let $\mathscr{F}$ be a family of meromorphic functions in a domain $D$, let $S_{1}=\left\{a_{1}, a_{2}, a_{3}\right\}, S_{2}=$ $\left\{b_{1}, b_{2}, b_{3}\right\}$, where $S_{1}$ and $S_{2}$ consist of finite complex numbers, let $k \geqslant 4$ and $q$ be two positive integers, and let a be any finite complex number. Suppose that for each $f \in \mathscr{F}$,

(i) $\bar{E}\left(S_{1}, f\right)=\bar{E}\left(S_{2},\left(f^{(k)}\right)^{q}\right)$; (ii) $f-a$ has zeros and poles with multiplicities at least $k$ and $k-1$, respectively,

then $\mathscr{F}$ is normal in $D$.

The following example due to Zhang et al [14] illustrates that the condition that the zeros of $f-$ $a$ have multiplicities at least $k>2$ is necessary in Theorem 1.

Example 1 We denote the unit disk $\{z \in \mathbb{C}:|z|<1\}$ by $\triangle$, let $\mathscr{F}=\left\{f_{n}\right\}$ be a family of meromorphic functions in $\triangle$, where $f_{n}=n\left(e^{w_{1} z}-e^{w_{2} z}\right), n=$ $1,2, \ldots, w_{1} \neq w_{2}, w_{1}^{k}=w_{2}^{k}=1$, and $k \geqslant 2$ be a positive integer. After a simple calculation, we obtain $f=f^{(k)}$ for each $f$, getting $\left|f^{\prime}(0)\right| /\left(1+|f(0)|^{2}\right)=$ $n\left(w_{1}-w_{2}\right) \rightarrow \infty$ for large $n$. According to Marty's normality criteria, we know $\mathscr{F}$ is not normal in $\triangle$.

\section{LEMMAS}

In order to prove our results, we need the following lemmas.

Lemma 1 ([15]) Let $\mathscr{F}$ be a family of meromorphic functions in the unit disk $\Delta$ with the property that for each $f \in \mathscr{F}$, both zeros and poles of $f-a$ are of multiplicities at least $k$ and $p$ respectively. Suppose that there exists a positive number $A>1$ such that $\left|f^{(k)}(z)\right| \leqslant A$ whenever $f(z)=0$. If $\mathscr{F}$ is not normal in $\Delta$, then for any real number $\alpha, p<\alpha \leqslant k$, there exists

(i) a sequence of complex numbers $z_{n} \rightarrow z_{0},\left|z_{n}\right|<r<1$,

(ii) a sequence of functions $f_{n} \in \mathscr{F}$,

(iii) a sequence of positive numbers $\rho_{n} \rightarrow 0$,

(iv) a real number $r, 0<r<1$,

such that $g_{n}(\xi)=\rho_{n}^{-\alpha} f_{n}\left(z_{n}+\rho_{n} \xi\right)$ converges spherically locally uniformly to a non-constant meromorphic function $g(\xi)$ in $\mathbb{C}$ such that $g^{\#}(\xi)=\frac{\left|g^{\prime}(\xi)\right|}{1+|g(\xi)|^{2}} \leqslant$ $g^{\#}(0)=k A+1$, where $g(\xi)$ has zeros and poles with multiplicities at least $k$ and $p$, respectively. Moreover, $g(\xi)$ is of order at most two.

Remark 2 If $\mathscr{F}$ is not normal at $z_{0} \in \Delta$, the above conclusion still holds when there exists points $z_{n}$ such that $z_{n} \rightarrow z_{0}$ for large $n$.

Lemma 2 Suppose that $f$ is a meromorphic function of finite order, $a_{1}, a_{2}$ and $a_{3}$ are three distinct finite complex numbers. If the number of zeros of $f$ is finite in $\mathbb{C}$ and $\left(f^{(k)}\right)^{q} \in S$ implies $f(z)=0$, where $S=\left\{a_{1}, a_{2}, a_{3}\right\}$, then $f$ is a rational function. 
Proof: By the second Nevanlinna fundamental theorem, we have

$$
\begin{aligned}
T\left(r,\left(f^{(k)}\right)^{q}\right) & \leqslant \sum_{i=1}^{3} \bar{N}\left(r, \frac{1}{\left(f^{(k)}\right)^{q}-a_{i}}\right)+S\left(r,\left(f^{(k)}\right)^{q}\right) \\
& \leqslant \bar{N}\left(r, \frac{1}{f}\right)+S\left(r,\left(f^{(k)}\right)^{q}\right) .
\end{aligned}
$$

Obviously,

$$
\begin{aligned}
T\left(r,\left(f^{(k)}\right)^{q}\right) & \leqslant q T\left(r, f^{(k)}\right) \\
& \leqslant q(k+1) T(r, f)+q S(r, f) .
\end{aligned}
$$

According to (1) and the assumption that $f$ is a meromorphic function of finite order, we see that $\left(f^{(k)}\right)^{q}$ is also a meromorphic function with finite order, and get

$$
S\left(r,\left(f^{(k)}\right)^{q}\right)=O(\log r) .
$$

By the condition that the number of zeros of $f(z)$ is finite in $\mathbb{C}$, we have

$$
\bar{N}\left(r, \frac{1}{f}\right)=O(\log r) .
$$

It follows from (2) and (3) that

$$
T\left(r,\left(f^{(k)}\right)^{q}\right) \leqslant O(\log r),
$$

which implies that $f$ is a rational function.

Lemma 3 ([3]) Let $f$ be a non-constant meromorphic function on the open complex plane, $k$ be a positive integer, then we get

$$
\begin{aligned}
& N\left(r, \frac{1}{f^{(k)}}\right) \leqslant T\left(r, f^{(k)}\right)-T(r, f)+N\left(r, \frac{1}{f}\right)+S(r, f), \\
& N\left(r, \frac{1}{f^{(k)}}\right) \leqslant N\left(r, \frac{1}{f}\right)+k \bar{N}(r, f)+S(r, f) .
\end{aligned}
$$

\section{PROOF OF Theorem 1}

Proof: Suppose that $\mathscr{F}$ is not normal in a domain $D$, without loss of generality, we assume that $\mathscr{F}$ is not normal at $z_{0} \in D$. Then we consider two cases.

Case 1. $a \in S_{1}$. Suppose that $a=a_{1}$, by Lemma 1 , there exists $z_{n} \rightarrow z_{0}, f_{n} \in \mathscr{F}$ and $\rho_{n} \rightarrow 0$ satisfying

$$
g_{n}(\zeta)=f_{n}\left(z_{n}+\rho_{n} \zeta\right)-a_{1} \rightarrow g(\zeta)
$$

uniformly on compact subsets of $\mathbb{C}$, where $g(\zeta)$ is a non-constant meromorphic function. Moreover, both zeros and poles of $g(\zeta)$ have multiplicities at least $k>2$.
Subcase 1.1 There exists $\zeta_{0} \in \mathbb{C}$ such that $g\left(\zeta_{0}\right)=0$.

Let $G_{n}(\zeta)=\rho_{n}^{-k}\left(f_{n}\left(z_{n}+\rho_{n} \zeta\right)-a_{1}\right)$. In the view of the condition, we know that zeros of $G_{n}(\zeta)$ have multiplicities at least $k$. Obviously, there is a neighbourhood $\left|\zeta-\zeta_{0}\right|<\delta\left(\zeta_{0}\right)$ such that $G_{n}(\zeta)$ is a holomorphic function. Now we claim that $\left\{G_{n}(\zeta)\right\}$ is not normal at $\zeta_{0}$. Indeed, if $\left\{G_{n}(\zeta)\right\}$ is normal at $\zeta_{0}$, we have $G_{n}\left(\zeta_{n}\right) \rightarrow G(\zeta)$ uniformly in $\left|\zeta-\zeta_{0}\right|<$ $\delta\left(\zeta_{0}\right)$ as $n \rightarrow \infty$, where $G(\zeta)$ is a holomorphic function. We have $g(\zeta) \not \equiv 0$ by the condition that $g(\zeta)$ is non-constant. By Hurwitz's theorem, we choose a sequence of points $\zeta_{n} \rightarrow \zeta_{0}$ such that $g_{n}\left(\zeta_{n}\right)=f_{n}\left(z_{n}+\rho_{n} \zeta_{n}\right)-a_{1}=0$ and $G_{n}\left(\zeta_{n}\right)=0$ for sufficiently large $n$. Clearly, there exists a deleted neighborhood (denoted by $\triangle^{\prime}\left(\zeta_{0}, \delta\left(\zeta_{0}\right)\right)=\{\zeta: 0<$ $\left.\left.\left|\zeta-\zeta_{0}\right|<\delta\left(\zeta_{0}\right)\right\}\right)$ of point $\zeta_{0}$ such that $g(\zeta) \neq 0$ for all $\zeta \in \triangle^{\prime}$, that is to say, $f_{n}\left(z_{n}+\rho_{n} \zeta\right) \neq a_{1}$ in $\triangle^{\prime}$, without loss of generality, suppose that $f_{n}\left(z_{n}+\right.$ $\left.\rho_{n} \zeta\right)=a_{2}$ in $\triangle^{\prime}$, then we know that $G_{n}(\zeta)=\infty$ in $\triangle^{\prime}$ as $n \rightarrow \infty$, which is conflicting.

$G_{n}(\zeta)=0$ is equal to $f_{n}\left(z_{n}+\rho_{n} \zeta\right)=a_{1}$. Based on the condition, we have $\left(f_{n}^{(k)}\left(z_{n}+\right.\right.$ $\left.\left.\rho_{n} \zeta\right)\right)^{q}=\left(G_{n}^{(k)}(\zeta)\right)^{q} \in S_{2}$ and choose $b_{i} \in S_{2}$ such that $\left(G_{n}^{(k)}(\zeta)\right)^{q}=b_{i}$, from which we know that $\left|G_{n}^{(k)}(\zeta)\right| \leqslant \sum_{i=1}^{3} \sqrt[q]{\left|b_{i}\right|}+k$ whenever $G_{n}(\zeta)=0$, where $i=1,2$, 3. Suppose that $A=\sum_{i=1}^{3} \sqrt[q]{\left|b_{i}\right|}+k$.

Combining with Lemma 1 , there exists $\zeta_{n} \rightarrow \zeta_{0}$, $\eta_{n} \rightarrow 0$ and a subsequence of functions $\left\{G_{n}\right\}$ such that

$$
F_{n}(\xi)=\eta_{n}^{-k} G_{n}\left(\zeta_{n}+\eta_{n} \xi\right) \rightarrow F(\xi)
$$

uniformly on any compact subset of $\mathbb{C}$. $F(\xi)$ is a non-constant meromorphic function such that $F^{\#}(\xi)=\left|F^{\prime}(\xi)\right| /\left(1+|F(\xi)|^{2}\right) \leqslant F^{\#}(0)=k A+1$. Moreover, $F(\xi)$ has zeros with multiplicities at least $k$ and $F(\xi)$ is of order at most 2 . We claim that

(a) The number of distinct zeros of $F(\xi)$ is finite in $\mathbb{C}$;

(b) $F(\xi)=0 \Longleftrightarrow\left(F^{(k)}(\xi)\right)^{q} \in S_{2}$.

Let $\zeta_{0}$ be a zero of $g(\zeta)$ with multiplicity $m$, we prove that $F(\xi)$ has at most $m$ different zeros. Otherwise, if $F(\xi)$ has $m+1$ distinct points $\xi_{1}, \xi_{2}, \ldots, \xi_{m+1}$ in $\mathbb{C}$ such that $F\left(\xi_{j}\right)=0$ for $1 \leqslant$ $j \leqslant m+1$. From Hurwitz's theorem and the fact that $F(\xi) \not \equiv 0$, there exists a sequence of points $\xi_{n_{j}} \rightarrow \xi_{j}$ satisfying $F_{n}\left(\xi_{n_{j}}\right)=0$, then we know that $f_{n}\left(z_{n}+\rho_{n}\left(\zeta_{n}+\eta_{n} \xi_{n_{j}}\right)\right)-a_{1}=0$ when $\zeta_{n}+\eta_{n} \xi_{n_{j}} \rightarrow \zeta_{0}$ for large $n$, where $j=1,2, \ldots, m+1$. We deduce that $\zeta_{0}$ is a zero of $g(\zeta)$ with multiplicity $m+1$ 
by Hurwitz's theorem, which contradicts the above hypothesis. Thus the proof of claim (a) is complete.

If $F\left(\xi_{0}\right)=0$, by Hurwitz's theorem and the condition $F(\xi) \not \equiv 0$, we can find points $\xi_{n} \rightarrow \xi_{0}$ such that $F_{n}\left(\xi_{n}\right)=0$, so is $f_{n}\left(z_{n}+\rho_{n}\left(\zeta_{n}+\eta_{n} \xi_{n}\right)\right)=a_{1}$. By the assumption that $\bar{E}\left(S_{1}, f\right)=\bar{E}\left(S_{2},\left(f^{(k)}\right)^{q}\right)$, we know that $\left(F_{n}^{(k)}\left(\xi_{n}\right)\right)^{q}=\left(f_{n}^{(k)}\left(z_{n}+\rho_{n}\left(\zeta_{n}+\eta_{n} \xi_{n}\right)\right)\right)^{q} \in$ $S_{2}$ and $\lim _{n \rightarrow \infty}\left(F_{n}^{(k)}\left(\xi_{n}\right)\right)^{q}=\left(F^{(k)}\left(\xi_{0}\right)\right)^{q} \in S_{2}$.

Thus we have proved $F(\xi)=0 \rightarrow\left(F^{(k)}(\xi)\right)^{q} \in$ $S_{2}$. On the other hand, if $\left(F^{(k)}\left(\xi_{0}\right)\right)^{q} \in S_{2}$, for a given point $\xi_{0} \in \mathbb{C}$, there exists $\left(F^{(k)}\left(\xi_{0}\right)\right)^{q}=b_{i}$, where $i=1,2,3$. First of all, we prove $\left(F^{(k)}(\xi)\right)^{q} \not \equiv b_{i}$. On the contrary, we suppose that $\left(F^{(k)}(\xi)\right)^{q} \equiv b_{i}$, from which we obtain that $F(\xi)$ is a polynomial with degree at most $k$, according to the fact that each zero of $F(\xi)$ is of multiplicity at least $k$, we claim that $F(\xi)$ is a polynomial of degree $k$ such that $F(\xi)=(k !) \sqrt[q]{b_{i}}\left(\xi-\xi_{0}\right)^{k}$. If $\sqrt[q]{b_{i}} \neq 0$, we have

$$
\begin{aligned}
& F^{\#}(0)=\frac{\left|F^{\prime}(0)\right|}{1+|F(0)|^{2}}=\frac{\frac{\sqrt[q]{\left|b_{i}\right|}}{(k-1) !}\left|\xi_{0}\right|^{k-1}}{1+\frac{\left(\sqrt[q]{\left|b_{i}\right|}\right)^{2}}{(k !)^{2}}\left|\xi_{0}\right|^{2 k}},
\end{aligned}
$$

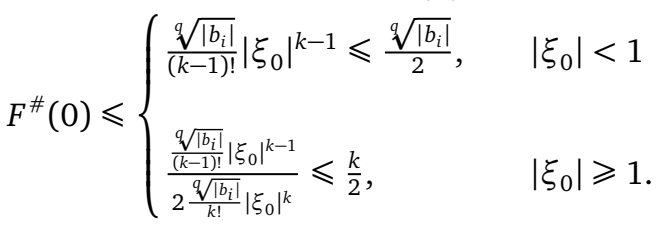

All in all, $F^{\#}(0) \leqslant \frac{\sqrt[q]{\left|b_{i}\right|}}{2}+\frac{k}{2}$. If $\sqrt[q]{\left|b_{i}\right|}=0$, the above inequality also holds, which contradicts the fact that $F^{\#}(0)=k A+1$. By Hurwitz's theorem and the condition that $\left(F^{(k)}(\xi)\right)^{q} \not \equiv b_{i}$, we choose a sequence of points $\xi_{n}$ satisfying $\xi_{n} \rightarrow \xi_{0}$, such that

$$
\left(F_{n}^{(k)}\left(\xi_{n}\right)\right)^{q}=\left(f_{n}^{(k)}\left(z_{n}+\rho_{n}\left(\zeta_{n}+\eta_{n} \xi_{n}\right)\right)\right)^{q}=b_{i} .
$$

Combining with the assumption

$$
\bar{E}\left(S_{1}, f\right)=\bar{E}\left(S_{2},\left(f^{(k)}\right)^{q}\right),
$$

we obtain $f_{n}\left(z_{n}+\rho_{n}\left(\zeta_{n}+\eta_{n} \xi_{n}\right)\right) \in S_{1}$. Next we prove that there exists a subsequence of $f_{n}$ such that $f_{n}\left(z_{n}+\rho_{n}\left(\zeta_{n}+\eta_{n} \xi_{n}\right)\right)=a_{1}$ if $n$ is sufficiently large. Otherwise, without loss of generality, suppose that $f_{n}\left(z_{n}+\rho_{n}\left(\zeta_{n}+\eta_{n} \xi_{n}\right)\right)=a_{2}$ for large $n$, we obtain $F\left(\xi_{0}\right)=\lim _{n \rightarrow \infty} \rho_{n}^{-k} \eta_{n}^{-k}\left(f_{n}\left(z_{n}+\rho_{n}\left(\zeta_{n}+\eta_{n} \xi_{n}\right)\right)-\right.$ $\left.a_{1}\right)=\lim _{n \rightarrow \infty} \rho_{n}^{-k} \eta_{n}^{-k}\left(a_{2}-a_{1}\right)=\infty$. This is inconsistent with the fact that $\left(F^{(k)}\left(\xi_{0}\right)\right)^{q}=b_{i}$. Hence, we get $F\left(\xi_{0}\right)=\lim _{n \rightarrow \infty} \rho_{n}^{-k} \eta_{n}^{-k}\left(f_{n}\left(z_{n}+\rho_{n}\left(\zeta_{n}+\eta_{n} \xi_{n}\right)\right)\right.$ $\left.a_{1}\right)=0$. This illustrates that $F(\xi)=0$ whenever $\left.F^{(k)}(\xi)\right)^{q} \in S_{2}$, thus claim (b) is proved.

By claims (a) and (b), and Lemma 2, we claim that $F(\xi)$ is a rational function. Next we prove that $F(\xi)$ is a polynomial.
Suppose that $F(\xi)$ is a non-constant rational function, but $F(\xi)$ is not a polynomial. Set $F=$ $\frac{H}{Q}$, both $H$ and $Q$ are relatively prime polynomials. Zeros of $H$ are $\alpha_{1}, \ldots, \alpha_{s}$ with multiplicities $m_{1}, \ldots, m_{s}$ respectively. Zeros of $Q$ are $\beta_{1}, \ldots, \beta_{t}$ with multiplicities $n_{1}, \ldots, n_{t}$, respectively. We get $m_{i} \geqslant k$ by the hypothesis that zeros of $F$ have multiplicities at least $k$, where $i=1,2, \ldots, s$. Let $M=\operatorname{deg} H=\sum_{i=1}^{s} m_{i}$ and $N=\operatorname{deg} Q=\sum_{j=1}^{t} n_{j}$. Write $\left(F^{(k)}\right)^{q}=\frac{\left(H_{k}\right)^{q}}{\left(Q_{k}\right)^{q}}$. After a simple calculation, we get $\operatorname{deg}\left(\left(Q_{k}\right)^{q}\right)=q(N+k t)$, thus

$$
2 q(N+k t) \log r \leqslant 2 T\left(r,\left(F^{(k)}\right)^{q}\right) .
$$

According to the second Nevanlinna fundamental theorem, we obtain

$$
\begin{aligned}
2 T\left(r,\left(F^{(k)}\right)^{q}\right) & \leqslant \sum_{i=1}^{3} \bar{N}\left(r, \frac{1}{\left(F^{(k)}\right)^{q}-b_{i}}\right)+\bar{N}(r, F)+O(1) \\
& \leqslant \bar{N}\left(r, \frac{1}{F}\right)+\bar{N}(r, F)+O(1) \\
& \leqslant(t+s) \log r+O(1)
\end{aligned}
$$

We find that $2 q(N+k t) \log r \leqslant(t+s) \log r+O(1)$ and $s \geqslant 2 q N+1$. By the condition that zeros of $F$ have multiplicities at least $k$, we have $M \geqslant k s \geqslant 2 q N k+$ $k>2 N$. After a simple calculation, we obtain

$$
\operatorname{deg}\left(\left(H_{k}\right)^{q}\right)-\operatorname{deg}\left(\left(Q_{k}\right)^{q}\right)=q(M-N-k)>0 .
$$

From (4), we have $\operatorname{deg}\left(\left(H_{k}\right)^{q}\right)=q((M-N-k)+N+$ $k t)=q(M+k(t-1)) \geqslant M$ and get

$$
\begin{aligned}
2 M \log r & \leqslant(t+s) \log r+O(1) \\
& \leqslant(M+N) \log r+O(1) .
\end{aligned}
$$

It follows from (5) that $M \leqslant N$, which is impossible.

Suppose that $F(\xi)=C_{p} \xi^{p}+C_{p-1} \xi^{p-1}+\cdots+$ $C_{k} \xi^{k}+C_{k-1} \xi^{k-1}+\cdots+C_{0}$, where $C_{i}, i=0,1, \ldots, p$, is a complex number, and $C_{p} \neq 0$. Combining with the condition that zeros of $F$ have multiplicities at least $k$, we obtain $p \geqslant k$.

By the second Nevanlinna fundamental theorem, we have

$$
\begin{aligned}
2 T\left(r,\left(F^{(k)}\right)^{q}\right) & \leqslant \sum_{i=1}^{3} \bar{N}\left(r, \frac{1}{\left(F^{(k)}\right)^{q}-b_{i}}\right)+\bar{N}(r, F)+O(1) \\
& \leqslant N\left(r, \frac{1}{F}\right)+O(1) \\
& =T(r, F)+O(1),
\end{aligned}
$$

from which we obtain $2 q(p-k) \log r \leqslant p \log r+O(1)$ and $k \leqslant p \leqslant\left(1+\frac{1}{2 q-1}\right) k \leqslant 2 k$.

Subcase 1.1.1 $p=k$. Then $F$ is a polynomial of degree $k$, so that $\left(F^{(k)}(\xi)\right)^{q} \equiv d$, where $d$ is a 
constant. If $d \in S_{2}$, we get $F(\xi) \equiv 0$ by claim (b), which is invalid. If $d \notin S_{2}$, we know that $F(\xi)$ is a non-zero constant from the fact that $F(\xi)$ is a polynomial, which contradicts the condition that $F$ is a non-constant meromorphic function.

Subcase 1.1.2 $p=2 k$. If $p=2 k$, we know that $F$ is a polynomial with degree $2 k$. We claim that $F$ has only two distinct zeros or only one zero by the condition that zeros of $F$ have multiplicity at least $k$.

Subcase 1.1.2.1 $F(\xi)$ has only one zero. From the second Nevanlinna fundamental theorem and claim (b),

$$
\begin{aligned}
2 T\left(r,\left(F^{(k)}\right)^{q}\right) \leqslant & \sum_{i=1}^{3} \bar{N}\left(r, \frac{1}{\left(F^{(k)}\right)^{q}-b_{i}}\right) \\
& +\bar{N}(r, F)+O(1) \\
\leqslant & N\left(r, \frac{1}{\left(F^{(k)}\right)^{q}}\right)+O(1) \\
= & T\left(r,\left(F^{(k)}\right)^{q}\right)+O(1),
\end{aligned}
$$

which is conflicting.

Subcase 1.1.2.2 $F(\xi)$ has only two distinct zeros. Write $F(\xi)=m\left(\xi-\xi_{0}\right)^{k}\left(\xi-\xi_{1}\right)^{k}$, where $m \neq 0$, $\xi_{0}$ and $\xi_{1}$ are distinct finite complex numbers. For any $i=1,2,3,\left(F^{(k)}(\xi)\right)^{q}-b_{i}$ has at least one zero, then we know that $F(\xi)$ has at least three distinct zeros by the fact that $\left(F^{(k)}(\xi)\right)^{q} \in S_{2} \rightarrow F(\xi)=0$, which is impossible.

Subcase $1.2 g(\zeta) \neq 0$. Suppose that there exists $\zeta_{0}$ satisfying $g\left(\zeta_{0}\right)=a_{2}-a_{1}$. Note that $g(\zeta) \not \equiv a_{2}-a_{1}$, by Hurwitz's theorem, we find a sequence $\zeta_{n}$ such that $g_{n}\left(\zeta_{n}\right)=a_{2}-a_{1}$ when $\zeta_{n} \rightarrow \zeta_{0}$, an equivalent statement is that $f_{n}\left(z_{n}+\right.$ $\left.\rho_{n} \zeta_{n}\right)=a_{2}$. Since $\bar{E}\left(S_{1}, f\right) \subseteq \bar{E}\left(S_{2},\left(f^{(k)}\right)^{q}\right)$, we obtain $\left(f_{n}^{(k)}\left(z_{n}+\rho_{n} \zeta_{n}\right)\right)^{q} \in S_{2}$ and find a subsequence of $\left\{f_{n}\right\}$ such that $\left(f_{n}^{(k)}\left(z_{n}+\rho_{n} \zeta_{n}\right)\right)^{q}=s$, and therefore $\left(g^{(k)}\left(\zeta_{0}\right)\right)^{q}=\lim _{n \rightarrow \infty}\left(g_{n}^{(k)}\left(\zeta_{n}\right)\right)^{q}=$ $\lim _{n \rightarrow \infty}\left(\rho_{n}^{k} f_{n}^{(k)}\left(z_{n}+\rho_{n} \zeta_{n}\right)\right)^{q}=\lim _{n \rightarrow \infty} \rho^{k q} s^{q}=0$, where $s \in S_{2}$. This illustrates that $\bar{E}\left(a_{2}-a_{1}, g\right) \subseteq$ $\bar{E}\left(0,\left(g^{(k)}\right)^{q}\right)$. In a similar fashion, we can prove $\bar{E}\left(a_{3}-a_{1}, g\right) \subseteq \bar{E}\left(0,\left(g^{(k)}\right)^{q}\right)$. By the second Nevanlinna fundamental theorem and the hypothesis that poles of $g$ are of multiplicities at least $k$ and Lemma 3,

$$
\begin{aligned}
2 T(r, g) \leqslant & \bar{N}(r, g)+\bar{N}\left(r, \frac{1}{g}\right) \\
& +\sum_{i=2}^{3} \bar{N}\left(r, \frac{1}{g-\left(a_{i}-a_{1}\right)}\right)+S(r, g) \\
\leqslant & \bar{N}(r, g)+\bar{N}\left(r, \frac{1}{\left(g^{(k))^{q}}\right.}\right)+S(r, g) \\
= & \bar{N}(r, g)+\bar{N}\left(r, \frac{1}{g^{(k)}}\right)+S(r, g),
\end{aligned}
$$

which gives

$$
\begin{aligned}
2 T(r, g) & \leqslant \frac{1}{k} N(r, g)+N\left(r, \frac{1}{g}\right)+k \bar{N}(r, g)+S(r, g) \\
& \leqslant\left(\frac{1}{k}+1\right) T(r, g)+S(r, g),
\end{aligned}
$$

which contradicts the fact that $k>2$.

Case 2. $a \notin S_{1}$. By Lemma 1 , there exists $z_{n} \rightarrow z_{0}, \rho_{n} \rightarrow 0$ and a subsequence of $f_{n} \in \mathscr{F}$ such that

$$
g_{n}(\zeta)=f_{n}\left(z_{n}+\rho_{n} \zeta\right)-a \rightarrow g(\zeta)
$$

uniformly on any compact subset of $\mathbb{C}$, where $g(\zeta)$ is a non-constant meromorphic function such that both zeros and poles of $g(\zeta)$ have multiplicities at least $k$. Similar to Subcase 1.2 , we have $\bar{E}\left(a_{i}-\right.$ $a, g) \subseteq \bar{E}\left(0,\left(g^{(k)}\right)^{q}\right), \quad i=1,2,3$. By the second Nevanlinna fundamental theorem and the fact that both zeros and poles of $g$ are of multiplicities at least $k$ and Lemma 3 , we get

$$
\begin{aligned}
3 T & (r, g) \\
& \leqslant \bar{N}(r, g)+\bar{N}\left(r, \frac{1}{g}\right)+\sum_{i=1}^{3} \bar{N}\left(r, \frac{1}{g-\left(a_{i}-a\right)}\right)+S(r g) \\
& =\bar{N}(r, g)+\bar{N}\left(r, \frac{1}{g}\right)+\bar{N}\left(r, \frac{1}{\left(g^{(k)) q}\right)+S(r, g)}\right. \\
& =\bar{N}(r, g)+\bar{N}\left(r, \frac{1}{g}\right)+\bar{N}\left(r, \frac{1}{g^{(k)}}\right)+S(r, g) \\
& \leqslant \frac{1}{k} N(r, g)+\frac{1}{k} N\left(r, \frac{1}{g}\right)+N\left(r, \frac{1}{g}\right)+N(r, g)+S(r, g) \\
& \leqslant\left(\frac{2}{k}+2\right) T(r, g)+S(r, g),
\end{aligned}
$$

which contradicts the fact that $k>2$. Theorem 1 is completely proved.

\section{PROOF OF Theorem 2}

Proof: Assume that $\mathscr{F}$ is not normal in a domain $D$, without loss of generality, we suppose that $\mathscr{F}$ is not normal at $z_{0} \in D$. By Lemma 1 , we choose $z_{n} \rightarrow z_{0}$, $f_{n} \in \mathscr{F}$ and $\rho_{n} \rightarrow 0$ such that

$$
g_{n}(\zeta)=f_{n}\left(z_{n}+\rho_{n} \zeta\right) \rightarrow g(\zeta)
$$

uniformly on compact subsets of $\mathbb{C}$. We know that at least one of $g(\zeta)-a_{i}$ must have zeros by the condition that $g(\zeta)$ is non-constant and Picard theorem, where $i=1,2,3$. Let $\zeta_{0}$ be a zero of $g(\zeta)-a_{1}$ with multiplicity $k$.

Write $G_{n}(\zeta)=\rho_{n}^{-k}\left(f_{n}\left(z_{n}+\rho_{n} \zeta\right)-a_{1}\right)$. According to the assumption, we claim that zeros of $G_{n}(\zeta)$ have multiplicities at least $k$. In the same manner as in the proof of Subcase 1.1 in Theorem 1, we can prove that $\left\{G_{n}(\zeta)\right\}$ is not normal at $\zeta_{0}$. Combining with the assumption that $\bar{E}\left(S_{1}, f\right)=\bar{E}\left(S_{2},\left(f^{(k)}\right)^{q}\right)$, we know that $\left|G_{n}^{(k)}(\zeta)\right| \leqslant \sum_{i=1}^{3} \sqrt[q]{\left|b_{i}\right|}+k$ whenever $G_{n}(\zeta)=0$. Suppose that $A=\sum_{i=1}^{3} \sqrt[q]{\left|b_{i}\right|}+k$. 
By Lemma 1 , there exists $\zeta_{n} \rightarrow \zeta_{0}, \eta_{n} \rightarrow 0$ and a sequence of $\left\{G_{n}\right\}$ such that $F_{n}(\xi)=\eta_{n}^{-k} G_{n}\left(\zeta_{n}+\eta_{n} \xi\right)$ which converges spherically locally uniformly to a non-constant meromorphic function $F(\xi)$ satisfying $F^{\#}(\xi)=\left|F^{\prime}(\xi)\right| /\left(1+|F(\xi)|^{2}\right) \leqslant F^{\#}(0)=k A+1$ on $\mathbb{C}$; moreover, zeros of $F(\xi)$ are of multiplicities at least $k$, and $F(\xi)$ is of order at most 2 .

Also as in the proof of Subcase 1.1 in Theorem 1, we claim that

(c) The number of distinct zeros of $F(\xi)$ is finite in $\mathbb{C}$;

(d) $F(\xi)=0 \Longleftrightarrow\left(F^{(k)}(\xi)\right)^{q} \in S_{2}$.

According to claims (c) and (d), and Lemma 2, we know that $F(\xi)$ is a rational function. In the similar fashion to the proof of Subcase 1.1 in Theorem 1, we claim that $F(\xi)$ is a polynomial.

Set $F(\xi)=C_{p} \xi^{p}+C_{p-1} \xi^{p-1}+\cdots+C_{k} \xi^{k}+$ $C_{k-1} \xi^{k-1}+\cdots+C_{0}$, where $C_{i}(i=0,1, \ldots, p)$ is a complex number, and $C_{p} \neq 0$. We get $p \geqslant k$ by the fact that zeros of $F(\xi)$ have multiplicities $\geqslant k$.

Next we can prove Theorem 2 by using the same argument as in Subcase 1.1 of Theorem 1.

\section{PROOF OF Theorem 3}

Proof: Suppose that $\mathscr{F}$ is not normal in a domain $D$, without loss of generality, we assume that $\mathscr{F}$ is not normal at $z_{0} \in D$. Then we consider two cases.

Case 1. $a \in S_{1}$. Suppose that $a=a_{1}$, by Lemma 1, there exists

(i) a sequence of complex numbers $z_{n} \rightarrow z_{0},\left|z_{n}\right|<$ $r<1$;

(ii) a sequence of functions $f_{n} \in \mathscr{F}$;

(iii) a sequence of positive numbers $\rho_{n} \rightarrow 0$, such that $g_{n}(\zeta)=f_{n}\left(z_{n}+\rho_{n} \zeta\right)-a_{1}$ converges uniformly with respect to the spherical metric to a nonconstant meromorphic function $g(\zeta)$ in $\mathbb{C}$. Moreover, $g(\zeta)$ is of order at most 2 , all of whose zeros and poles have multiplicities at least $k \geqslant 2$ and $k+1$, respectively.

Subcase 1.1 There exists $\zeta_{0} \in \mathbb{C}$ such that $g\left(\zeta_{0}\right)=0$. Set $G_{n}(\zeta)=\rho_{n}^{-k}\left(f_{n}\left(z_{n}+\rho_{n} \zeta\right)-a_{1}\right)$. By the condition, we know that zeros of $G_{n}(\zeta)$ have multiplicities at least $k \geqslant 2$. Obviously, there is a neighbourhood $\left|\zeta-\zeta_{0}\right|<\delta\left(\zeta_{0}\right)$ such that $G_{n}(\zeta)$ is a holomorphic function.

Also as in the proof of Subcase 1.1 in Theorem 1, we claim that $\left\{G_{n}(\zeta)\right\}$ is not normal at $\zeta_{0}$ and $\left|G_{n}^{(k)}(\zeta)\right| \leqslant \sum_{i=1}^{3} \sqrt[q]{\left|b_{i}\right|}+k$ whenever $G_{n}(\zeta)=0, i=$ $1,2,3$. Suppose that $A=\sum_{i=1}^{3} \sqrt[q]{\left|b_{i}\right|}+k$.

Combining with Lemma 1 , there exists $\zeta_{n} \rightarrow$ $\zeta_{0}, \eta_{n} \rightarrow 0$ and a subsequence of functions $\left\{G_{n}\right\}$ such that $F_{n}(\xi)=\eta_{n}^{-k} G_{n}\left(\zeta_{n}+\eta_{n} \xi\right)$ converges locally uniformly to a non-constant meromorphic function $F(\xi)$ such that $F^{\#}(\xi)=\left|F^{\prime}(\xi)\right| /\left(1+|F(\xi)|^{2}\right) \leqslant$ $F^{\#}(0)=k A+1$ on any compact subset of $\mathbb{C}$, where $F(\xi)$ has zeros with multiplicities at least $k$ and $F(\xi)$ is of order at most 2 . We claim that

(e) The number of distinct zeros of $F(\xi)$ is finite in $\mathbb{C}$;

(f) $F(\xi)=0 \Longleftrightarrow\left(F^{(k)}(\xi)\right)^{q} \in S_{2}$.

By claims (e) and (f) and, Lemma 2, we claim that $F(\xi)$ is a rational function. In a similar fashion to the proof of Subcase 1.1 in Theorem 1, we claim that $F(\xi)$ is a polynomial.

Next, the proof of Subcase 1.1 in Theorem 3 is completely similar with the proof of Subcase 1.1 in Theorem 1.

Subcase $1.2 g(\zeta) \neq 0$. If there exists $\zeta_{0}$ such that $g\left(\zeta_{0}\right)=a_{2}-a_{1}$. By the fact that $g(\zeta) \not \equiv a_{2}-$ $a_{1}$ and Hurwitz's theorem, we find a sequence $\zeta_{n}$ such that $g_{n}\left(\zeta_{n}\right)=a_{2}-a_{1}$ when $\zeta_{n} \rightarrow \zeta_{0}$, which means that $f_{n}\left(z_{n}+\rho_{n} \zeta_{n}\right)=a_{2}$. We obtain $\left(f_{n}^{(k)}\left(z_{n}+\right.\right.$ $\left.\left.\rho_{n} \zeta_{n}\right)\right)^{q} \in S_{2}$ and find a subsequence of $\left\{f_{n}\right\}$ such that $\left(f_{n}^{(k)}\left(z_{n}+\rho_{n} \zeta_{n}\right)\right)^{q}=s$ by the condition that $\bar{E}\left(S_{1}, f\right) \subseteq \bar{E}\left(S_{2},\left(f^{(k)}\right)^{q}\right)$, and therefore $\left(g^{(k)}\left(\zeta_{0}\right)\right)^{q}=$ $\left.\lim _{n \rightarrow \infty}\left(g_{n}^{(k)}\left(\zeta_{n}\right)\right)^{q}=\lim _{n \rightarrow \infty} \rho_{n}^{k} f_{n}^{(k)}\left(z_{n}+\rho_{n} \zeta_{n}\right)\right)^{q}=$ $\lim _{n \rightarrow \infty} \rho^{k q} s^{q}=0$, where $s \in S_{2}$. This illustrates that $\bar{E}\left(a_{2}-a_{1}, g\right) \subseteq \bar{E}\left(0,\left(g^{(k)}\right)^{q}\right)$. In a similar fashion, we can prove $\bar{E}\left(a_{3}-a_{1} g\right) \subseteq \bar{E}\left(0,\left(g^{(k)}\right)^{q}\right)$. By the second Nevanlinna fundamental theorem and the hypothesis that poles of $g$ are of multiplicities at least $k+1$, and Lemma 3 , it is known that

$$
\begin{aligned}
2 T(r, g) \leqslant & \bar{N}(r, g)+\bar{N}\left(r, \frac{1}{g}\right) \\
& +\sum_{i=2}^{3} \bar{N}\left(r, \frac{1}{g-\left(a_{i}-a_{1}\right)}\right)+S(r, g) \\
\leqslant & \bar{N}(r, g)+\bar{N}\left(r, \frac{1}{\left(g^{(k)}\right)}\right)+S(r, g) \\
= & \bar{N}(r, g)+\bar{N}\left(r, \frac{1}{g^{(k)}}\right)+S(r, g) \\
\leqslant & \frac{1}{k+1} N(r, g)+N\left(r, \frac{1}{g}\right)+k \bar{N}(r, g)+S(r, g) \\
\leqslant & \left(\frac{1}{k+1}+\frac{k}{k+1}\right) T(r, g)+S(r, g) \\
= & T(r, g)+S(r, g),
\end{aligned}
$$

which is conflicting.

Case 2. $a \notin S_{1}$. By Lemma 1 , there exists $z_{n} \rightarrow z_{0}, \rho_{n} \rightarrow 0$ and a subsequence of $f_{n} \in \mathscr{F}$ such that $g_{n}(\zeta)=f_{n}\left(z_{n}+\rho_{n} \zeta\right)-a$ converges locally uniformly to a non-constant meromorphic function $g(\zeta)$ on any compact subset of $\mathbb{C}$, where both zeros and poles of $g(\zeta)$ have multiplicities at least $k$ and 
$k+1$, respectively. It is similar to Subcase 1.2 , we have $\bar{E}\left(a_{i}-a, g\right) \subseteq \bar{E}\left(0,\left(g^{(k)}\right)^{q}\right), i=1,2,3$.

By the second Nevanlinna fundamental theorem and the fact that both zeros and poles of $g$ are of multiplicities at least $k$ and $k+1$, respectively, and Lemma 3, we get

$$
\begin{aligned}
3 T(r, g) \leqslant & \bar{N}(r, g)+\bar{N}\left(r, \frac{1}{g}\right) \\
& +\sum_{i=1}^{3} \bar{N}\left(r, \frac{1}{g-\left(a_{i}-a\right)}\right)+S(r, g) \\
= & \bar{N}(r, g)+\bar{N}\left(r, \frac{1}{g}\right)+\bar{N}\left(r, \frac{1}{\left(g^{(k))^{q}}\right.}\right)+S(r, g) \\
= & \bar{N}(r, g)+\bar{N}\left(r, \frac{1}{g}\right)+\bar{N}\left(r, \frac{1}{g(k)}\right)+S(r, g) \\
\leqslant & \frac{1}{k+1} N(r, g)+\frac{1}{k} N\left(r, \frac{1}{g}\right)+N\left(r, \frac{1}{g}\right) \\
& +\frac{k}{k+1} N(r, g)+S(r, g) \\
\leqslant & \left(\frac{1}{k}+2\right) T(r, g)+S(r, g),
\end{aligned}
$$

which contradicts the fact that $k \geqslant 2$. Theorem 3 is proved completely.

\section{PROOF OF Theorem 4}

Proof: If $\mathscr{F}$ is not normal in a domain $D$, without loss of generality, we suppose that $\mathscr{F}$ is not normal at $z_{0} \in D$. Next, we consider two cases.

Case 1. $a \in S_{1}$. Assume that $a=a_{1}$, by Lemma 1 , there exists $z_{n} \rightarrow z_{0}, f_{n} \in \mathscr{F}$ and $\rho_{n} \rightarrow 0$ satisfying

$$
g_{n}(\zeta)=f_{n}\left(z_{n}+\rho_{n} \zeta\right)-a_{1} \rightarrow g(\zeta)
$$

uniformly on compact subsets of $\mathbb{C}$, where $g(\zeta)$ has zeros and poles with multiplicities at least $k \geqslant$ 4 and $k-1$, respectively, $g(\zeta)$ is a non-constant meromorphic function of order at most 2 .

Subcase 1.1 There exists $\zeta_{0} \in \mathbb{C}$ such that $g\left(\zeta_{0}\right)=0$. Write $G_{n}(\zeta)=\rho_{n}^{-k}\left(f_{n}\left(z_{n}+\rho_{n} \zeta\right)-a_{1}\right)$. We know that $G_{n}(\zeta)$ has zeros with multiplicities at least $k \geqslant 4$ by the condition. It is clear to find a neighbourhood $\left|\zeta-\zeta_{0}\right|<\delta\left(\zeta_{0}\right)$ such that $G_{n}(\zeta)$ is a holomorphic function.

In a similar fashion to the proof of Subcase 1.1 in Theorem 1, we claim that $\left\{G_{n}(\zeta)\right\}$ is not normal at $\zeta_{0}$ and $\left|G_{n}^{(k)}(\zeta)\right| \leqslant \sum_{i=1}^{3} \sqrt[q]{\left|b_{i}\right|}+k$ whenever $G_{n}(\zeta)=$ $0, i=1,2,3$. Suppose that $A=\sum_{i=1}^{3} \sqrt[q]{\left|b_{i}\right|}+k$.

By Lemma 1, there exists $\zeta_{n} \rightarrow \zeta_{0}, \eta_{n} \rightarrow 0$ and a subsequence of functions $\left\{G_{n}\right\}$ such that

$$
F_{n}(\xi)=\eta_{n}^{-k} G_{n}\left(\zeta_{n}+\eta_{n} \xi\right) \rightarrow F(\xi)
$$

uniformly on any compact subset of $\mathbb{C}$. $F(\xi)$, whose zeros have multiplicities at least $k \geqslant 4$, is a nonconstant meromorphic function such that $F^{\#}(\xi)=$
$\left|F^{\prime}(\xi)\right| /\left(1+|F(\xi)|^{2}\right) \leqslant F^{\#}(0)=k A+1$, and $F(\xi)$ is of order at most 2 . We claim that

(g) The number of distinct zeros of $F(\xi)$ is finite in $\mathbb{C}$;

(h) $F(\xi)=0 \Longleftrightarrow\left(F^{(k)}(\xi)\right)^{q} \in S_{2}$.

We claim that $F(\xi)$ is a rational function according to claims $(\mathrm{g})$ and $(\mathrm{h})$, and Lemma 2 . In a similar fashion to the proof of Subcase 1.1 in Theorem 1, we claim that $F(\xi)$ is a polynomial.

Next, also as in the proof of Subcase 1.1 in Theorem 1, we can prove Subcase 1.1 in Theorem 4.

Subcase $1.2 g(\zeta) \neq 0$. Without loss of generality, we assume that there exists $\zeta_{0}$ satisfying $g\left(\zeta_{0}\right)=a_{2}-a_{1}$. Combining with Hurwitz's theorem and the fact that $g(\zeta)$ is non-constant, there exists a sequence $\zeta_{n}$ such that $g_{n}\left(\zeta_{n}\right)=a_{2}-a_{1}$ and $f_{n}\left(z_{n}+\rho_{n} \zeta_{n}\right)=a_{2}$ for $\zeta_{n} \rightarrow \zeta_{0}$. We get $\left(f_{n}^{(k)}\left(z_{n}+\rho_{n} \zeta_{n}\right)\right)^{q} \in S_{2}$ and choose a subsequence of $\left\{f_{n}\right\}$ such that $\left(f_{n}^{(k)}\left(z_{n}+\rho_{n} \zeta_{n}\right)\right)^{q}=s$ by the condition, so $\left(g^{(k)}\left(\zeta_{0}\right)\right)^{q}=\lim _{n \rightarrow \infty}\left(g_{n}^{(k)}\left(\zeta_{n}\right)\right)^{q}=$ $\lim _{n \rightarrow \infty}\left(\rho_{n}^{k} f_{n}^{(k)}\left(z_{n}+\rho_{n} \zeta_{n}\right)\right)^{q}=\lim _{n \rightarrow \infty} \rho^{k q} s^{q}=0$, where $s \in S_{2}$. This illustrates that $\bar{E}\left(a_{2}-a_{1}, g\right) \subseteq$ $\bar{E}\left(0,\left(g^{(k)}\right)^{q}\right)$. In a similar fashion, we can prove $\bar{E}\left(a_{3}-a_{1}, g\right) \subseteq \bar{E}\left(0,\left(g^{(k)}\right)^{q}\right)$. By the second Nevanlinna fundamental theorem and the hypothesis that poles of $g$ are of multiplicities at least $k-1$ and Lemma 3, it is known that

$$
\begin{aligned}
2 T(r, g) \leqslant & \bar{N}(r, g)+\bar{N}\left(r, \frac{1}{g}\right) \\
& +\sum_{i=2}^{3} \bar{N}\left(r, \frac{1}{g-\left(a_{i}-a_{1}\right)}\right)+S(r, g) \\
\leqslant & \bar{N}(r, g)+\bar{N}\left(r, \frac{1}{\left(g^{(k)} q\right.}\right)+S(r, g) \\
= & \bar{N}(r, g)+\bar{N}\left(r, \frac{1}{g^{(k)}}\right)+S(r, g) \\
\leqslant & \frac{1}{k-1} N(r g)+N\left(r, \frac{1}{g}\right)+\frac{k}{k-1} N(r, g)+S(r, g) \\
= & \left(\frac{2}{k-1}+1\right) T(r, g)+S(r, g),
\end{aligned}
$$

which contradicts the fact that $k \geqslant 4$.

Case 2. $a \notin S_{1}$. According to Lemma 1 , there exists $z_{n} \rightarrow z_{0}, \rho_{n} \rightarrow 0$ and a subsequence of $f_{n} \in \mathscr{F}$ such that

$$
g_{n}(\zeta)=f_{n}\left(z_{n}+\rho_{n} \zeta\right)-a \rightarrow g(\zeta)
$$

uniformly on any compact subset of $\mathbb{C}$, where $g(\zeta)$ is a non-constant meromorphic function such that both zeros and poles of $g(\zeta)$ have multiplicities at least $k \geqslant 4$ and $k-1$, respectively. By using the same argument as in Subcase 1.2, we have $\bar{E}\left(a_{i}-a, g\right) \subseteq$ 
$\bar{E}\left(0,\left(g^{(k)}\right)^{q}\right), i=1,2,3$. By the second Nevanlinna fundamental theorem and the fact that both zeros and poles of $g$ are of multiplicities at least $k$ and $k-1$, respectively, and Lemma 3 , we get

$$
\begin{aligned}
3 T(r, g) \leqslant & \bar{N}(r, g)+\bar{N}\left(r, \frac{1}{g}\right) \\
& +\sum_{i=1}^{3} \bar{N}\left(r, \frac{1}{g-\left(a_{i}-a\right)}\right)+S(r, g) \\
= & \bar{N}(r, g)+\bar{N}\left(r, \frac{1}{g}\right)+\bar{N}\left(r, \frac{1}{\left(g^{(k)}\right)}\right)+S(r, g) \\
= & \bar{N}(r, g)+\bar{N}\left(r, \frac{1}{g}\right)+\bar{N}\left(r, \frac{1}{g^{(k)}}\right)+S(r, g) \\
\leqslant & \frac{1}{k-1} N(r, g)+\frac{1}{k} N\left(r, \frac{1}{g}\right)+N\left(r, \frac{1}{g}\right) \\
& +\frac{k}{k-1} N(r, g)+S(r, g) \\
< & \left(\frac{2+k}{k-1}+1\right) T(r, g)+S(r, g) \\
\leqslant & 3 T(r, g)+S(r, g)
\end{aligned}
$$

which contradicts the fact that $k \geqslant 4$. Theorem 4 is proved completely.

\section{OPEN QUESTION}

In Theorem 1, if $S_{1}$ and $S_{2}$ consist of holomorphic functions, and we replace $a$ with $a(z)$ being any holomorphic function, it is natural to ask:

Open question: Let $\mathscr{F}$ be a family of meromorphic functions in a domain $D$, let $S_{1}=\left\{a_{1}(z), a_{2}(z), a_{3}(z)\right\}, S_{2}=\left\{b_{1}(z), b_{2}(z), b_{3}(z)\right\}$, where $S_{1}$ and $S_{2}$ consist of holomorphic functions, let $k>2$ and $q$ be two positive integers, and let $a(z)$ be any holomorphic function. Suppose that for each $f \in \mathscr{F}$,

(i) $\bar{E}\left(S_{1}, f\right)=\bar{E}\left(S_{2},\left(f^{(k)}\right)^{q}\right)$;

(ii) both zeros and poles of $f-a(z)$ have multiplicities at least $k$, then $\mathscr{F}$ is normal in $D$.

Acknowledgements: This work was supported by the Funding Scheme Cultivating Innovation Ability for the graduate students of Guangzhou University (2018GDJCD28), NSF of China (11701111) and NSF of Guangdong
Province (2016A030310257). Visiting Scholar Program of Chern Institute of Mathematics at Nankai University when the authors worked as visiting scholars. The authors would like to express their hearty thanks to Chern Institute of Mathematics for very comfortable research environment.

\section{REFERENCES}

1. Laine I (1993) Nevanlinna Theory and Complex Differential Equations, Walter de Gruyter, Berlin.

2. Yang L (1993) Value Distribution Theory, Science Press, Beijing.

3. Gu YX, Pang XC, Fang ML (2007) Theory of Normal Families and Its Applications, Science Press, Beijing.

4. Ahlfors L (1979) Complex Analysis, 3rd edn, McGraw-Hill, New York.

5. Schiff J (1993) Normal Families, Springer-Verlag, Berlin.

6. Pang XC (2000) Normality of a family of meromorphic functions and normal function. Chin Annal Math 21, 601-604.

7. Yi HX, Yang CC (2003) The Uniqueness Theory of Meromorphic Function, Kluwer Academic Publishers, Dordrecht.

8. Hayman WK (1964) Meromorphic Functions, Clarendon Press, Oxford.

9. Schwick W (1992) Sharing values and normality. Arch Math 59, 50-54.

10. Fang ML (1996) A note on sharing values and normality. Math Res 29, 29-32.

11. Fang ML, Zalcman L (2004) A note on normality and shared values. Aust Math Soc 76, 141-150.

12. Pang XC, Zalcman L (2000) Normality and shared values. Ark Math 38, 171-182.

13. Liu XJ, Pang XC (2007) Shared values and normality. Acta Math Sin 52, 409-412.

14. Zhang H, Xie D, Zhang QD (2011) Normal criteria concerning shared values of meromorphic function. Acta Math Sci 31, 1290-1294.

15. Pang XC, Zalcman L (2000) Normal families and shared values. Bull London Math Soc 32, 325-331. 